\title{
Sulforaphane induces p53-deficient SW480 cell apoptosis via the ROS-MAPK signaling pathway
}

\author{
HAI LAN, HONGYIN YUAN and CONGYAO LIN
}

Department of Oncology, Zhongnan Hospital of Wuhan University, Wuhan, Hubei 430071, P.R. China

Received July 31, 2016; Accepted May 8, 2017

DOI: $10.3892 / \mathrm{mmr} .2017 .7558$

\begin{abstract}
Sulforaphane (SFN) has been revealed to inhibit the growth and induce apoptosis of cancer cells. However, the detailed anticancer effects of SFN on p53-deficient colon cancer cells has yet to be clearly elucidated. The present study employed p53-deficient SW480 cells to establish an $\mathrm{SFN}$-induced in vitro model of apoptosis. The critical events leading to apoptosis were then evaluated in SFN-treated p53-deficient SW480 cells, by performing an MTT assay, flow cytometry, western blotting and ELISA. The results demonstrated that SFN at concentrations of 5, 10, 15 and $20 \mu \mathrm{M}$ induced mitochondria-associated cell apoptosis, which was further confirmed by disruption of the mitochondrial membrane potential, an increase in the $\mathrm{Bax} / \mathrm{Bcl}-2$ ratio, as well as activation of caspase-3, -7 and -9. In addition, SFN-induced apoptosis was associated with an increase in the generation of reactive oxygen species (ROS), and the activation of extracellular signal-regulated kinases (Erk) and p38 mitogen-activated protein kinases. However, SFN did not induce expression of the p53 family member, p73. SFN-induced apoptosis was subsequently confirmed to be ROS-dependent and associated with Erk/p38, as the specific inhibitors for ROS, phosphorylated (p)-Erk and p-p38, completely or partially attenuated the SFN-induced reduction in SW480 cell viability. In addition, the results demonstrated that even at the lowest concentrations $(5 \mu \mathrm{M}), \mathrm{SFN}$ increased the sensitivity of $\mathrm{p} 53$-proficient
\end{abstract}

Correspondence to: Professor Congyao Lin, Department of Oncology, Zhongnan Hospital of Wuhan University, 169 Donghu Road, Wuhan, Hubei 430071, P.R. China

E-mail: congyaolinwh2016@126.com

Abbreviations: SFN, sulforaphane; MAPKs, mitogen-activated protein kinases; Erk, extracellular signal-regulated kinases; FBS, fetal bovine serum; ITCs, isothiocyanates; FITC, fluorescein isothiocyanate; PI, propidium iodide; MMP, mitochondrial membrane potential; ROS, reactive oxygen species; $\mathrm{H}_{2}$ DCF-DA, dichlorodihydrofluorescein diacetate; MDR, multi-drug resistance; JNK, c-Jun N-terminal kinase; SAPK, stress-activated protein kinase; CPDD, cisplatin

Key words: sulforaphane, reactive oxygen species, SW480 cells, extracellular signal-regulated kinases, p38
HCT-116 cells to cisplatin. In conclusion, the results suggest that SFN may induce apoptosis in p53-deficient SW480 cells via p53/p73-independent and ROS-Erk/p38-dependent signaling pathways.

\section{Introduction}

A previous study reported that individuals who regularly consume cruciferous vegetables demonstrate a lower risk of developing cancer, including breast, prostate and hepatic cancer (1). Cruciferous vegetables are a rich source of isothiocyanates (ITCs), which have been demonstrated to exhibit chemopreventive effects in animal cancer models $(1,2)$. Among ITCs, sulforaphane (SFN; $\mathrm{C}_{6} \mathrm{H}_{11} \mathrm{~S}_{2} \mathrm{NO}$ ) is abundant in broccoli and has been demonstrated to induce growth arrest and apoptosis of tumor cells in a variety of experimental cancer models $(2,3)$. SFN exerts its anticancer effects on tumor cells by regulating a number of cell signaling pathways including Kelch-like $\mathrm{ECH}$-associated protein 1-nuclear factor (NF)-erythroid 2-related factor 2 signaling, mitogen-activated protein kinase (MAPK) and NF-кB signaling (4-6). Therefore, SFN is considered to be a conceptually promising agent for cancer therapy. However, the detailed molecular mechanisms underlying the effects of SFN in colon cancer have yet to be fully elucidated.

Colorectal tumors first arise in the large intestine or the rectum, and is the third and second most common cancer in males and females, respectively $(7,8)$. This progressive cancer arises from the accumulation of mutations in tumor suppressor genes and oncogenes, which disrupt the homeostatic balance between cell proliferation and apoptosis (9). The p53 tumor suppressor gene serves important roles in the regulation of cell proliferation and apoptosis (10-12). p53 is activated by a number of intracellular and extracellular stressors, including irradiation, DNA damaging agents or reactive oxygen species (ROS) (13-15). Deficiencies in the function of p53 are critical events during the development of tumors and resistance to anticancer therapies $(16,17)$. It has been estimated that $\sim 29 \%$ of colorectal cancers harbor p53 gene mutations (18). Deficiencies in $\mathrm{p} 53$ function attenuate $\mathrm{p} 53$-mediated cell apoptosis and may lead to multi-drug resistance (MDR) $(19,20)$. An increasing body of evidence has indicated that $\mathrm{p} 53$-mutant or p53-null cancer cells are more likely to be resistant to different cytotoxic drugs; particularly to chemotherapy and radiation therapy in the clinic, as these therapies target cells primarily by inducing apoptosis $(19,21)$. Therefore, anticancer agents 
that induce cancer cell death in a p53-independent manner may be promising candidates for preventing MDR induced by p53 deficiency.

ROS serve important roles in the induction of apoptosis under normal physiological and abnormal pathological conditions $(22,23)$. Tumor cells exhibit increased ROS generation when compared to normal cells due to their vigorous metabolic activities (24-26). Increased ROS production in tumor cells increases their susceptibility to oxidative stress-induced apoptosis, which may be exploited by selective anticancer agents (27). A number of known natural compounds, including Aloin, curcumin and resveratrol, increase ROS levels, which leads to cancer cell death (28). A previous study demonstrated that SFN generate ROS, which subsequently induces apoptosis in a number of cancer cells. However, the specific role of ROS generation in response to SFN exposure in p53-deficient human colon cancer cells has not been fully elucidated (29).

In addition to p53, MAPK family members are activated by ROS (30-32). MAPKs, including extracellular signal-regulated kinase (Erk), c-Jun N-terminal kinase (JNK) and p38, are activated in response to diverse stimuli, and serve important roles in mammalian cells by regulating cell apoptosis, differentiation, migration and proliferation (33). JNK, also known as the stress-activated protein kinase, is predominantly activated by different stressors via the phosphorylation of the $\mathrm{NH}_{2}$-terminus of the c-Jun transcription factor. In the majority of cases, p38 MAPK signaling has been demonstrated to promote apoptosis or facilitate cell survival depending on the cell type and stimulus (28). However, Erk activation commonly induces cell proliferation or differentiation in response to cell stressors $(34,35)$.

A previous study revealed that SFN increases the generation of intracellular ROS, leading to mitochondrial dysfunction and human cancer cell apoptosis (36); however, the effects of SFN on p53-deficient colon cancer cells is currently unknown. In the present study, it was hypothesized that SFN induces apoptosis in association with ROS generation and mitochondrial dysfunction. To verify this hypothesis, the effect of SFN on the mitochondrial membrane potential (MMP), and ROS and apoptosis levels was determined using p53-deficient SW480 cells. The results demonstrated that, SFN increased ROS generation, which subsequently lead to activation of Erk and p38 MAPKs and apoptosis induction via the mitochondrial-dependent apoptotic pathway.

\section{Materials and methods}

Reagents and antibodies. Dulbecco's modified Eagle's medium (DMEM), RPMI-1640 medium and fetal bovine serum (FBS) were supplied by Hyclone; GE Healthcare Life Sciences (Logan, UT, USA). MTT, SFN and Cisplatin (CPDD; cat. no. P4394) were obtained from Sigma-Aldrich; Merck KGaA (Darmstadt, Germany). The ROS, phosphorylated (p)-p38 and Erk inhibitors [ammonium pyrrolidinedithiocarbamate (APDC), SB203580 and SCH772984], the caspase 3, 7, 8 and 9 activity assay kits (cat. nos. C1116, C1136, C1151 and C1157), ROS Assay kit (cat. no. S0033), Lipid Peroxidation Malondialdehyde (MDA) Assay kit (cat. no. S0131), Total Superoxide Dismutase (SOD) Assay kit with water-soluble tetrazolium salt (WST-8; cat. no. S0101) and Cellular
Glutathione Peroxidase (GSH-Px) Assay kit (cat. no. S0056), were all supplied by Beyotime Institute of Biotechnology (Nanjing, China). Protein isolation and bicinchoninic acid (BCA) protein quantification kits were supplied by Bio-Rad Laboratories, Inc. (Hercules, CA, USA). The annexin V-fluorescein isothiocyanate (FITC)/propidium iodide (PI) apoptosis detection kit was obtained from Multi Sciences (Lianke) Biotech Co, Ltd. (Hangzhou, China). Primary antibodies against B-cell lymphoma (Bcl-2; cat. no. BS4024), Bcl-2-associated X protein (Bax; cat. no. MB9013), p73 (cat. no. BS2452), p53 upregulated modulator of apoptosis (PUMA; cat. no. BS2922), phorbol-12-myristate-13-acetate-induced protein 1 (NOXA; cat. no. BS6214) and $\gamma \mathrm{H} 2 \mathrm{AX}$ (cat. no. BS4760) were obtained from Bioworld Technology, Inc. (St. Louis Park, MN, USA). Primary antibodies against p-Erk 1/2 (cat. no. sc-23759-R), p-p38 (cat. no. sc-7973) and p-JNK (cat. no. sc-135642) were obtained from Santa Cruz Biotechnology, Inc. (Dallas, TX, USA). The primary antibody against $\beta$-actin (cat. no. BA2359) was supplied by Wuhan Boster Biological Technology, Ltd. (Wuhan, China). IRDye-conjugated anti-rabbit (cat. no. 611-645-122) and anti-mouse (cat. no. 610-142-002) secondary antibodies were obtained from Rockland Immunochemicals, Inc. (Limerick, PA, USA).

Cell culture. The human colon adenocarcinoma cell line, SW480, and the human colon cancer cell line, HCT-116, were obtained from The Cell Bank of Type Culture Collection of Chinese Academy of Sciences (Shanghai, China). SW480 and HCT-116 cells were cultured in DMEM and RPMI-1640 medium, respectively, supplemented with $10 \%$ (v/v) FBS, and incubated at $37^{\circ} \mathrm{C}$ in a humidified $5 \% \mathrm{CO}_{2}$ incubator.

Cell viability assay. Cell viability was determined using an MTT assay. Briefly, SW480 or HCT-116 cells were plated into 96-well culture plates at an optimal density of $5 \times 10^{3}$ cells/well. SW480 cells were incubated for $24 \mathrm{~h}$ until cells had reached $90 \%$ confluence, and were pretreated with or without $0.1 \mu \mathrm{M}$ of the specific inhibitors against p38, Erk and ROS for $1 \mathrm{~h}$, before they were treated with $0,1,5,10,15$ and $20 \mu \mathrm{M} \mathrm{SFN}$ for 3, 6, 12,24 or $48 \mathrm{~h}$, respectively. HCT-116 cells were incubated for $24 \mathrm{~h}$ until cells had reached $90 \%$ confluence before they were treated with $0,1,5,10$ or $15 \mu \mathrm{M} \mathrm{SFN}$, together with 0 or $10 \mu \mathrm{M}$ CPDD, for $24 \mathrm{~h}$. The supernatant was replaced with $100 \mu \mathrm{l}$ $(500 \mu \mathrm{g} / \mathrm{ml})$ MTT solution and incubated for a further $4 \mathrm{~h}$. The supernatant was removed and $150 \mu \mathrm{l}$ dimethyl sulfoxide was added to dissolve the formazan crystals. The optical density was measured at $570 \mathrm{~nm}$ for each sample using a microtiter plate reader (BioTek Instruments, Inc, Winooski, VT, USA). The results are expressed as the percentage viability of the control at each time point $(100 \%)$.

Determination of cell apoptosis. Cell apoptosis was analyzed using an annexin V-FITC and PI double-staining method. Briefly, SW480 cells were seeded in 6-well plates at $3 \times 10^{5}$ cells/well and incubated for $24 \mathrm{~h}$ until they had reached $90 \%$ confluence. Cells were then treated with $0,1,5,10,15$ and $20 \mu \mathrm{M}$ SFN for $24 \mathrm{~h}$, and adherent cells were washed with PBS, collected and re-suspended in a binding buffer containing 1\% (v:v) annexin V-FITC and 2\% (v:v) PI. Cells 
were then incubated in the dark at room temperature for $20 \mathrm{~min}$. Apoptotic cells were quantified using a flow cytometer (BD Biosciences, Franklin Lakes, NJ, USA). A minimum of $1 \times 10^{5}$ cells for each sample were collected for flow cytometry analysis, recorded on a dot plot, and the percentage of apoptotic cells in the population was calculated using ModFit software (version 2.0.0; Verity Software House, Inc, Topsham, ME, USA).

Western blot analysis. The protein expression levels of p73, PUMA, NOXA, p-p38, p-Erk, p-JNK, Bcl-2 and Bax were measured in SW480 cells, and the expression levels of $\gamma \mathrm{H} 2 \mathrm{AX}$ were measured in HCT-116 cells by western blot analysis. $\beta$-actin was used as a loading control for these cell lines. Briefly, SW480 or HCT-116 cells were seeded in $25 \mathrm{~cm}^{2}$ cell culture dishes $\left(1 \times 10^{6}\right.$ cells/dish) and incubated for $24 \mathrm{~h}$ until they reached $90 \%$ confluence. SW480 and HCT-116 cells were treated with $0,1,5,10,15$ and/or $20 \mu \mathrm{M} \mathrm{SFN}$, together with 0 or $10 \mu \mathrm{M}$ CPDD, for $24 \mathrm{~h}$, and the adherent cells were washed with PBS, collected and lysed using a protein isolation kit (Bio-Rad Laboratories, Inc.) according to the manufacturer's instructions. Protein concentrations were quantified using a BCA protein quantification kit (Bio-Rad Laboratories, Inc.) according to the manufacturer's instructions. Whole cell lysates were mixed with a loading buffer (cat. no. P0015; Beyotime Institute of Biotechnology), and a total of $40 \mu \mathrm{g}$ cell lysate was boiled at $95^{\circ} \mathrm{C}$ for $5 \mathrm{~min}$ and then separated by $8-12 \%$ SDS-PAGE. The protein bands were transferred to polyvinylidene fluoride (PVDF) membranes according to manufacturer's instructions (EMD Millipore, Billerica, MA, USA). The PVDF membranes were blocked in Tris-buffered saline with $0.1 \%$ Tween 20 (TBST) containing $5 \%$ non-fat skim milk at $4^{\circ} \mathrm{Cfor} 1 \mathrm{~h}$, rinsed with TBST and probed with primary antibodies at $4^{\circ}$ Covernight (dilution, 1:500). Membranes were then incubated with the corresponding IRDye-conjugated secondary antibodies (dilution, 1:2,000) at room temperature for $1 \mathrm{~h}$. Membranes were visualized using an Odyssey Infrared Imaging System (LI-COR Biosciences, Lincoln, NE, USA). The relative optical densities of the target proteins were analyzed using Quantity One software (v4.62, Bio-Rad Laboratories, Inc.). The expression levels of target proteins were normalized to that of $\beta$-actin internal controls.

Caspase activity assay. The activities of caspase-3, -7, -8 and -9 were determined using caspase activity kits (Beyotime Institute of Biotechnology) according to the manufacturer's instructions. Briefly, SW480 cells were seeded in $25 \mathrm{~cm}^{2}$ cell culture dishes (1x10\% dish) and incubated for $24 \mathrm{~h}$ until they reached $90 \%$ confluence. Cells were then treated with $0,1,5$, 10,15 and $20 \mu \mathrm{M}$ SFN for $24 \mathrm{~h}$. Adherent cells were washed with PBS, collected and lysed with Radioimmunoprecipitation (RIPA) Lysis Buffer (cat. no. P0013C; Beyotime Institute of Biotechnology). The protein concentration was determined using a BCA protein quantification kit (Bio-Rad Laboratories, Inc.). Equal quantities $(4 \mu \mathrm{g} / \mathrm{ml})$ of whole cell lysates were then incubated with the corresponding caspase substrates to a final concentration of $2 \mathrm{mM}$ at $37^{\circ} \mathrm{C}$ for $2 \mathrm{~h}$. Samples were measured using a EL800 Microplate Reader (BioTek Instruments, Inc.) at $488 \mathrm{~nm}$ excitation and $525 \mathrm{~nm}$ emission.
Measurement of MMP. The MMP was analyzed by staining SW480 cells using a JC-1 probe (C2005, Beyotime Institute of Biotechnology), according to the manufacturer's instructions. Briefly, SW480 cells were seeded in 96-well plates at $3 \times 10^{3}$ cells/well and incubated for $24 \mathrm{~h}$ until reaching $90 \%$ confluence. The cells were subsequently treated with $0,1,5$, 10,15 and $20 \mu \mathrm{M}$ SFN for $24 \mathrm{~h}$. The cells were then incubated with $100 \mu \mathrm{l} \mathrm{JC}-1$ staining solution $(5 \mu \mathrm{g} / \mathrm{ml})$ at $37^{\circ} \mathrm{C}$ for $20 \mathrm{~min}$, before cells were rinsed twice with phosphate-buffered saline. The MMP was measured by determining the relative quantity of dual emissions from mitochondrial JC-1 monomers or aggregates using a fluorescence EL800 Microplate Reader (BioTek Instruments, Inc.) at excitation and emission wavelengths of 488 and $525 \mathrm{~nm}$, respectively. Mitochondrial depolarization is expressed as the red/green fluorescence intensity ratio, according to manufacturer's recommendations.

Measurement of intracellular ROS and MDA generation. The production of ROS was analyzed by staining SW480 cells with a dichlorodihydrofluorescein diacetate $\left(\mathrm{H}_{2} \mathrm{DCF}-\mathrm{DA}\right)$ probe. Briefly, the SW480 cells were seeded in 96-well plates at $3 \times 10^{3}$ cells/well and incubated for $24 \mathrm{~h}$ until they reached $90 \%$ confluence. The cells were then treated with $0,1,5,10,15$ and $20 \mu \mathrm{M}$ SFN for $24 \mathrm{~h}$, before the supernatant was removed. Cells were subsequently incubated with $\mathrm{H}_{2}$ DCF-DA solution $(2 \mu \mathrm{M})$ for $30 \mathrm{~min}$ at room temperature and rinsed three times using FBS-free medium. The fluorescence intensity was measured using a fluorescent ELISA reader (BioTek Instruments, Inc.) at excitation and emission wavelengths of 488 and $525 \mathrm{~nm}$, respectively.

MDA generation was detected using a lipid peroxidation MDA assay kit based on thiobarbituric acid (TBA) method. SW480 cells were seeded, cultured and treated with SFN using the same aforementioned methods, before the supernatant was subsequently collected and incubated with TBA (at a dilution of $1: 200$ ) at $100^{\circ} \mathrm{C}$ for $15 \mathrm{~min}$. The remaining procedures were performed according to the manufacturer's instructions (Beyotime Institute of Biotechnology). The MDA content in the supernatant was measured at $532 \mathrm{~nm}$ using an EL800 Microplate Reader (BioTek Instruments, Inc.).

Antioxidant activity assay. SOD activities in total cell lysates were examined using a total SOD assay kit based on the WST- 8 method (Beyotime Institute of Biotechnology), according to the manufacturer's instructions. Briefly, SW480 cells were seeded in $25 \mathrm{~cm}^{2}$ cell culture dishes (1x10\%/dish) and incubated for $24 \mathrm{~h}$ until they reached $90 \%$ confluence. Cells were then treated with $0,1,5,10,15$ and $20 \mu \mathrm{M}$ SFN for $24 \mathrm{~h}$. Subsequently, the adherent cells were washed with PBS, collected and lysed with RIPA Lysis Buffer (Beyotime Institute of Biotechnology) according to the buffer manufacturer's instructions. The protein concentration was determined using a BCA protein quantification kit (Bio-Rad Laboratories, Inc.). Equal quantities of whole cell lysates $(4 \mu \mathrm{g} / \mu \mathrm{l})$ were incubated with WST-8 solution (at a dilution of $1: 200$ ) at $37^{\circ} \mathrm{C}$ for $30 \mathrm{~min}$, and the remaining procedures were performed according to the manufacturer's instructions (Beyotime Institute of Biotechnology). SOD activity in total cell lysates was measured at $550 \mathrm{~nm}$ using the EL800 Microplate Reader (BioTek Instruments, Inc.). 
GSH-Px activities in total cell lysates were examined using a cellular GSH-Px assay kit. Briefly, SW480 cells were seeded in $25 \mathrm{~cm}^{2}$ cell culture dishes and incubated for $24 \mathrm{~h}$ until they reached $90 \%$ confluence. The cells were then treated with 0 , $1,5,10,15$ and $20 \mu \mathrm{M} \mathrm{SFN}$ for $24 \mathrm{~h}$ before the adherent cells were washed with PBS, collected and lysed with the RIPA Lysis Buffer (Beyotime Institute of Biotechnology), according to the buffer manufacturer's instructions. Protein concentrations were quantified using a BCA protein quantification kit. Equal quantities of whole cell lysates $(2 \mu \mathrm{g} / \mu \mathrm{l})$ were incubated with the assay kit buffer (GPx detection working buffer, at a $1: 1$ dilution) at $37^{\circ} \mathrm{C}$ for $30 \mathrm{~min}$, and the remaining procedures were performed according to the manufacturer's instructions. The GSH-Px activity in total cell lysates was measured at $412 \mathrm{~nm}$ using the EL800 Microplate Reader (BioTek Instruments, Inc.).

Statistical analysis. The results are presented as the mean \pm standard deviation of three independent experiments and the statistical significance was analyzed by one-way analysis of variance followed by a post-hoc Dunnett's test. Analysis was performed using SPSS 16.0 (SPSS, Inc, Chicago, IL, USA). P $<0.05$ was considered to indicate a statistically significant difference.

\section{Results}

SFN inhibits SW480 cell viability. To investigate the effects of SFN on SW480 cell growth, the cells were treated with increasing concentrations $(0,1,5,10,15$ and $20 \mu \mathrm{M})$ of SFN for 3, 6, 12, 24 and $48 \mathrm{~h}$, and an MTT assay was performed to assess cell viability. As shown in Fig. 1A, SFN inhibited SW480 cell proliferation in a dose- and time-dependent manner. When compared with the untreated control group at each time point, the viability of cells treated with $5,10,15$ and $20 \mu \mathrm{M}$ SFN were significantly inhibited $(\mathrm{P}<0.05$ and $\mathrm{P}<0.01$ ). In addition, the viability of cells treated with $20 \mu \mathrm{M}$ SFN at 3,6, 12, 24 and $48 \mathrm{~h}$ were significantly reduced when compared with controls, with 70.2, 66.3, 51.5, 38.6 and 20.1\% viabilities, respectively ( $\mathrm{P}<0.01$; Fig. 1A). However, compared with the untreated controls, no significant differences in the viability of cells treated with $1 \mu \mathrm{M} \mathrm{SFN}$ for 3, 6, 12, 24 and $48 \mathrm{~h}$ were observed. Therefore, for all subsequent assays, cells were exposed to SFN for $24 \mathrm{~h}$ as it exhibited an appropriate inhibiting ratio for cell viability.

In order to determine whether the SFN-induced inhibition of SW480 cell viability was due to the induction of apoptosis, the proportion of apoptotic cells in the population was analyzed by flow cytometry using FITC and PI double-staining. As shown in Fig. 1B and summarized in Fig. 1C, exposure of SW480 cells to 5, 10, 15 and $20 \mu \mathrm{M}$ SFN for $24 \mathrm{~h}$, significantly increased the percentage of apoptotic cells when compared with the untreated controls $(\mathrm{P}<0.01)$; the percentage of apoptotic cells in the $20 \mu \mathrm{M}$ SFN-treated group reached $71.9 \%$. However, $1 \mu \mathrm{M} \mathrm{SFN}$ failed to induce a significant difference in the proportion of apoptotic cells (Fig. 1B and C). These results indicated that SW480 cells may undergo apoptosis following treatment with SFN, and suggested an association between the induction of apoptosis and the inhibition of cell viability.

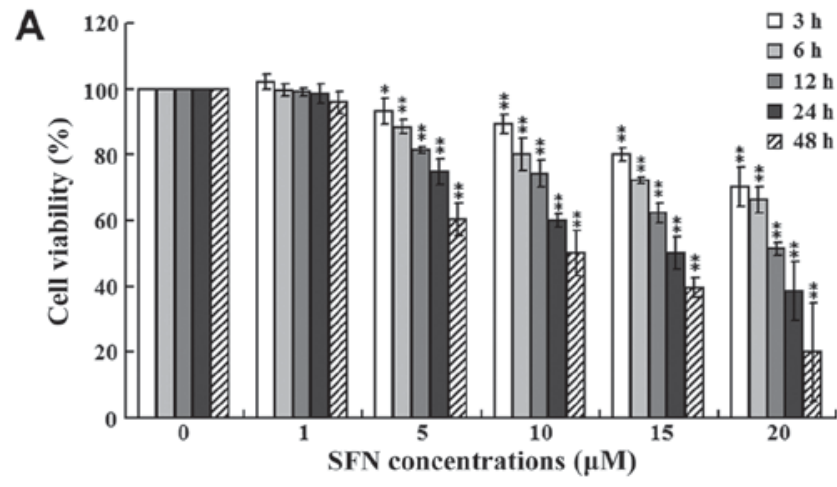

B
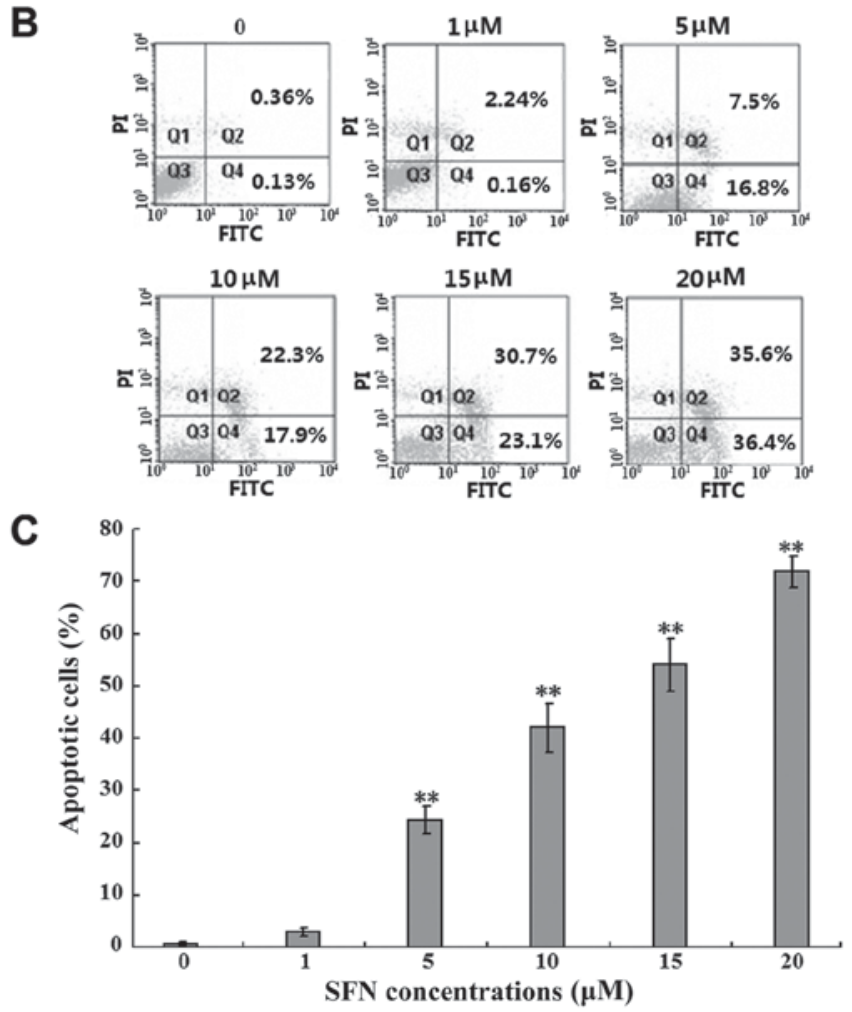

Figure 1. Effects of SFN on SW480 cell viability and apoptosis. (A) SW480 cells were treated with $0,1,5,10,15$ and $20 \mu \mathrm{M} \mathrm{SFN}$ for $3,6,12,24$ and $48 \mathrm{~h}$, and cell viability was assessed using an MTT assay. The viability of cells without SFN treatment was considered as $100 \%$, and the results from three independent experiments are shown $\left({ }^{*} \mathrm{P}<0.05\right.$ and ${ }^{* *} \mathrm{P}<0.01$ vs. $0 \mu \mathrm{M} \mathrm{SFN}$ at each time point). (B) SW480 cells were treated with $0,1,5,10,15$ and $20 \mu \mathrm{M}$ SFN for $24 \mathrm{~h}$. The percentage of apoptotic cells was then examined by flow cytometry analysis. The percentage of apoptotic cells was calculated as the sum of Q2 and Q4. (C) Quantification of the percentage of apoptotic cells from three independent experiments are shown $\left({ }^{* *} \mathrm{P}<0.01\right.$ vs. $\left.0 \mu \mathrm{M} \mathrm{SFN}\right)$. SFN, sulforaphane; Q, quadrant.

SFN-induced apoptosis is p73-independent and is associated with the intrinsic apoptotic signaling pathway. To determine whether SFN-induced SW480 cell apoptosis is p73-dependent, the protein expression levels of p73, as well as its downstream effectors, PUMA and NOXA, were detected. As shown in Fig. 2A, p73 protein was not detected in the untreated control group nor in the SFN-treated groups. By contrast, PUMA and NOXA were expressed in the SW480 cells; however, no significant differences were observed between the untreated and SFN-treated groups (Fig. 2A).

The present study further investigated the role of caspase- 3 , $-7,-8$, and -9 in SFN-induced SW480 cell apoptosis. As shown 

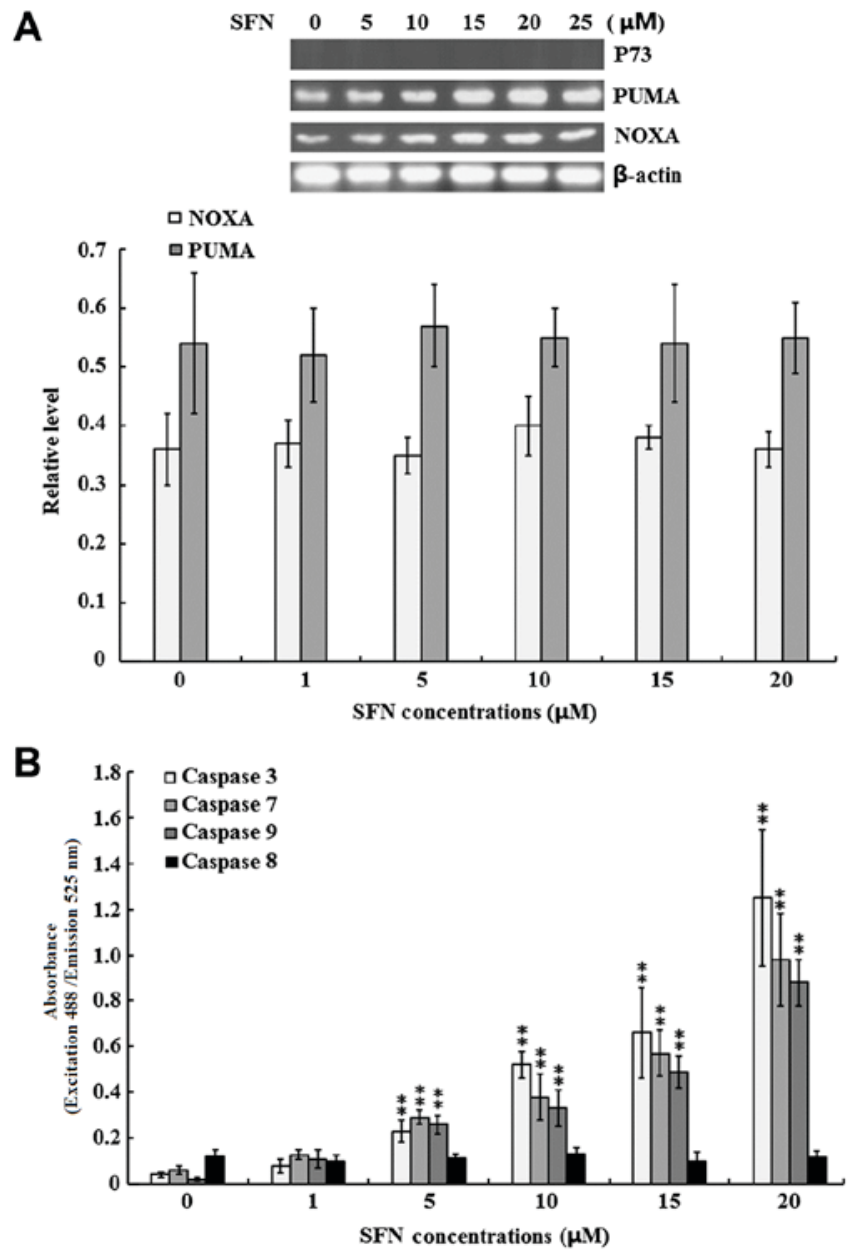

Figure 2. Effect of SFN on p73 expression and caspase enzyme activities. SW480 cells were treated with $0,1,5,10,15$ and $20 \mu \mathrm{M}$ SFN for $24 \mathrm{~h}$. (A) The protein expression levels of p73, PUMA and NOXA were examined by western blotting. Quantified band densities are summarized below the images of the bands, and target protein expression levels were normalized to that of b-actin. (B) The activity of caspase-3, -7, -8 and -9 was determined using specific ELISA kits. The results of three independent experiments are shown. ${ }^{* *} \mathrm{P}<0.01$ vs. $0 \mu \mathrm{M}$ SFN. SFN, sulforaphane; PUMA, p53 upregulated modulator of apoptosis; NOXA, phorbol-12-myristate-13-acetate-induced protein 1; ELISA, enzyme-linked immunosorbent assay.

in Fig. 2B, the activity of the intrinsic apoptotic executioners, caspase-3, -7 and -9 , were significantly increased in the 5, 10, 15 and $20 \mu \mathrm{M}$ SFN-treated groups when compared with the untreated control group $(\mathrm{P}<0.01)$. By contrast, the activity of the extrinsic apoptotic executioner, caspase 8, was not significantly induced under the same conditions (Fig. 2B).

SFN-induced apoptosis is associated with p38 and Erk activation. In addition to p53 and p73, the MAPK signaling pathway regulates cellular proliferation and apoptosis under cell stress conditions (30). Therefore, the present study investigated whether SFN-induced apoptosis was regulated by the activation of Erk, JNK and p38. As shown in Fig. 3A, the protein expression levels of p-Erk and p-p38 were significantly increased in 5, 10, 15 and $20 \mu \mathrm{M}$ SFN-treated groups when compared with the untreated control group $(\mathrm{P}<0.05$ and $\mathrm{P}<0.01)$.

A previous study indicated that the anti-apoptotic functions of $\mathrm{Bcl}-2$ and the pro-apoptotic functions of Bax are regulated by MAPKs (37). Therefore, the present study examined the protein expression levels of Bax and Bcl-2 in SFN-treated SW480 cells. As shown in Fig. 3B, the protein expression levels of Bcl-2 were significantly decreased in the 5, 10, 15 and $20 \mu \mathrm{M}$ SFN-treated groups when compared with the untreated control group $(\mathrm{P}<0.05$ and $\mathrm{P}<0.01)$; however, no significant differences in the level of Bax protein were detected in the SFN-treated groups compared with the controls. Accordingly, a significant increase in the $\mathrm{Bax} / \mathrm{Bcl}-2$ ratio was observed when compared with the untreated controls $(\mathrm{P}<0.05$ and $\mathrm{P}<0.01$; Fig. 3C). Previous studies have demonstrated that an increased $\mathrm{Bax} / \mathrm{Bcl}-2$ ratio induces alterations in the mitochondrial permeability transition; an event which subsequently leads to disruption of the MMP $(38,39)$. Therefore, the present study investigated whether SFN exposure altered the MMAs shown in Fig. 3D, exposure to 5, 10, 15 and $20 \mu \mathrm{M}$ SFN induced significant mitochondrial depolarization when compared with the untreated group, as indicated by the decreased red/green fluorescence ratio $(\mathrm{P}<0.01)$.

SFN induces oxidative stress in SW480 cells. ROS are known to function as messengers that activate MAPKs (26). Therefore, the present study investigated whether SFN induced SW480 cell apoptosis via the generation of ROS. As shown in Fig. 4A, exposure to 5, 10, 15 and $20 \mu \mathrm{M} \mathrm{SFN}$ significantly increased ROS production when compared with the untreated group $(\mathrm{P}<0.01)$. This result was further confirmed by the significant increase in MDA production following exposure of cells to 1, 5, 10, 15 and $20 \mu \mathrm{M} \mathrm{SFN}$ when compared with untreated controls (Fig. 4B). MDA production is the direct consequence of ROS production (40). The activity of the anti-oxidative enzymes, SOD and GSH-Px, were not significantly altered following exposure to increasing concentrations of SFN (Fig. 4C and D).

SFN-induced apoptosis is ROS dependent and involves p38 and Erk cell signaling pathways. To discriminate between the different roles of ROS, p38 and Erk in SFN-induced apoptosis, SFN-treated SW480 cells were treated with specific inhibitors against p38, Erk and ROS. First, the effects of the inhibitors on ROS generation were investigated. As shown in Fig. 5A, the ROS inhibitor, APDC, completely attenuated the SFN-induced production of ROS. By contrast, treatment with the p38 and Erk inhibitors (SB203580 and SCH772984, respectively), demonstrated no marked effects on the SFN-induced production of ROS. The effects of the inhibitors on cell viability were then evaluated. As presented in Fig. 5B, treatment of cells with the ROS inhibitor, APDC, completely attenuated the SFN-mediated inhibition of SW480 cell viability. By contrast, treatment with the p38 inhibitor (SB203580), and the Erk inhibitor (SCH772984) partially attenuated the SFN-mediated inhibition of SW480 cell proliferation. These results indicated that SFN increased ROS generation and induced cell apoptosis via the p38 and Erk cell signaling pathways.

SFN sensitizes p53 proficient HCT-116 cells to CPDD-induced apoptosis by increasing DNA damage. Finally, the present study evaluated the ability of SFN to potentiate the inhibitory action of CPDD against the proliferation of $\mathrm{p} 53$-proficient HCT-116 colon cancer cells. The effects of SFN on 
A

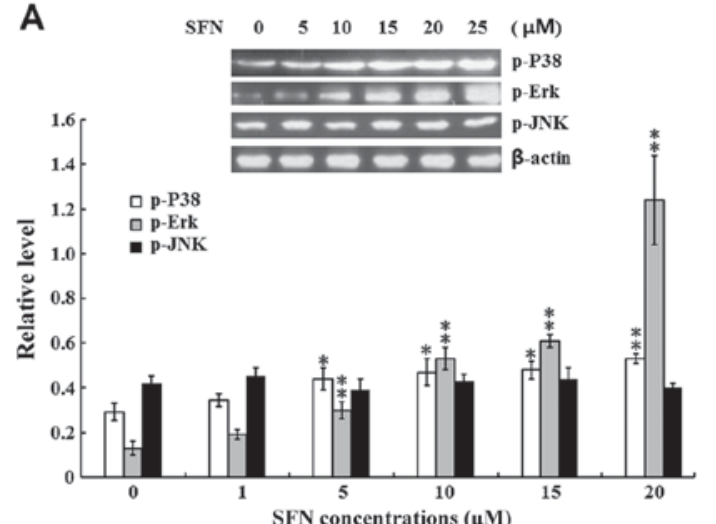

C

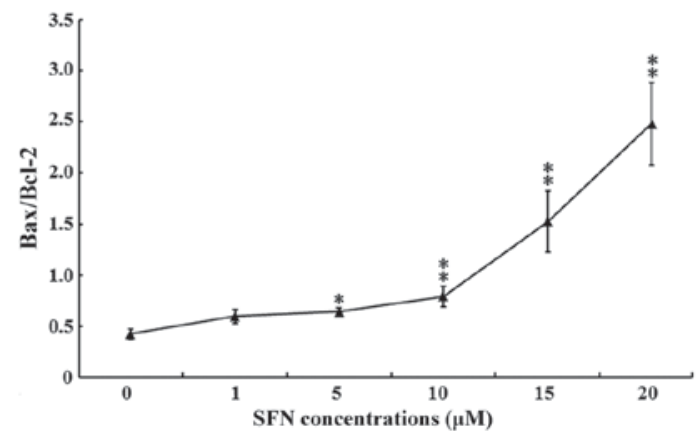

B

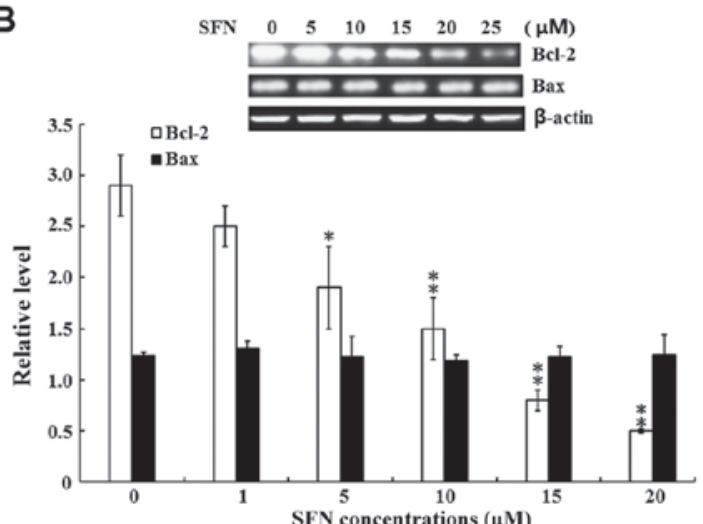

D

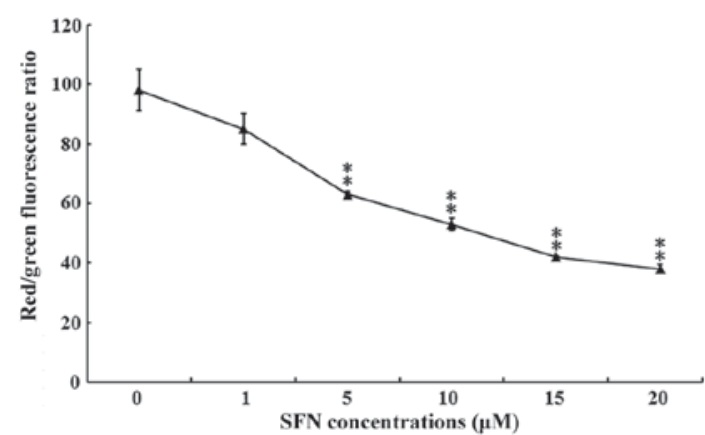

Figure 3. Effect of SFN on MAPKs and intrinsic apoptotic signaling pathways. SW480 cells were treated with $0,1,5,10,15$ and $20 \mu \mathrm{M}$ SFN for $24 \mathrm{~h}$. (A) The protein expression levels of (A) p-p38, p-Erk and p-JNK and (B) Bcl-2 and Bax were examined by western blotting, and the quantified results are summarized below the images of the protein bands. Target protein expression levels were normalized to that of b-actin. (C) The Bax/Bcl-2 ratio. (D) The MMP was examined using a MMP assay kit with a JC-1 probe. The results of three independent experiments are shown. ${ }^{*} \mathrm{P}<0.05$ and ${ }^{* *} \mathrm{P}<0.01$ vs. $0 \mu \mathrm{M}$ SFN. SFN, sulforaphane; MAPK, mitogen-activated protein kinases; p-, phosphorylated; Erk, extracellular signal-regulated kinases; JNK, c-Jun N-terminal kinases; Bcl-2, B-cell lymphoma-2; Bax, Bcl-2-associated protein X; MMP, mitochondrial membrane potential.
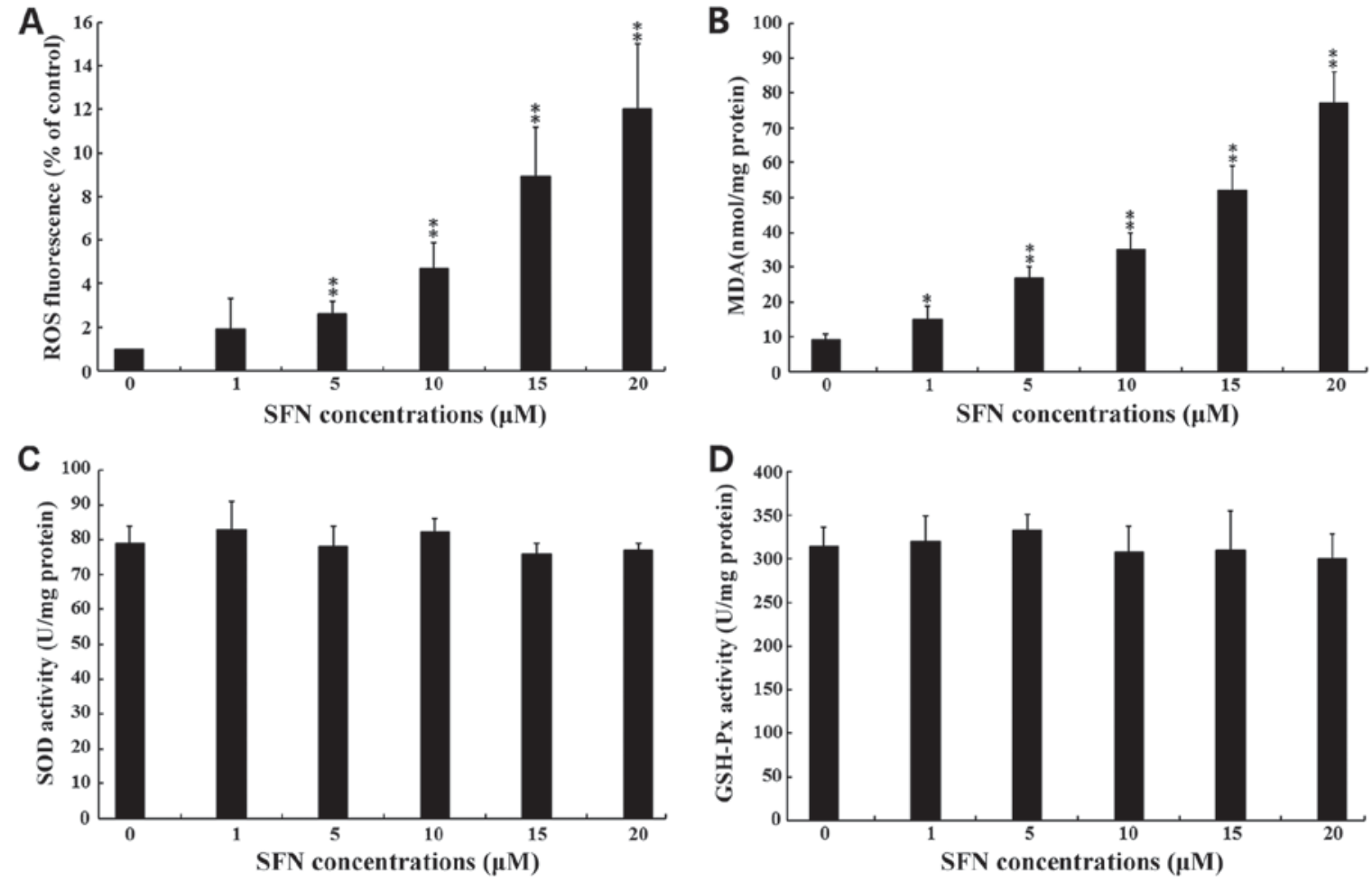

Figure 4. Effect of SFN on oxidative stress levels. SW480 cells were treated with $0,1,5,10,15$ and $20 \mu \mathrm{M}$ SFN for 24 . (A) Intracellular ROS production was examined using a ROS assay kit. (B) Intracellular MDA levels were examined using a lipid peroxidation MDA assay kit. (C) SOD activities in total cell lysates were examined using a total SOD assay kit using water-soluble tetrazolium salt-8. (D) GSH-Px activities in total cell lysates were examined using a cellular GSH-Px assay kit. For all experiments, the results of three independent experiments are shown. " $\mathrm{P}<0.05$ and ${ }^{* * *} \mathrm{P}<0.01$ vs. $0 \mu \mathrm{M}$ SFN. SFN, sulforaphane; ROS, reactive oxygen species; MDA, malondialdehyde; SOD, superoxide dismutase; GSH-Px, glutathione peroxidase. 

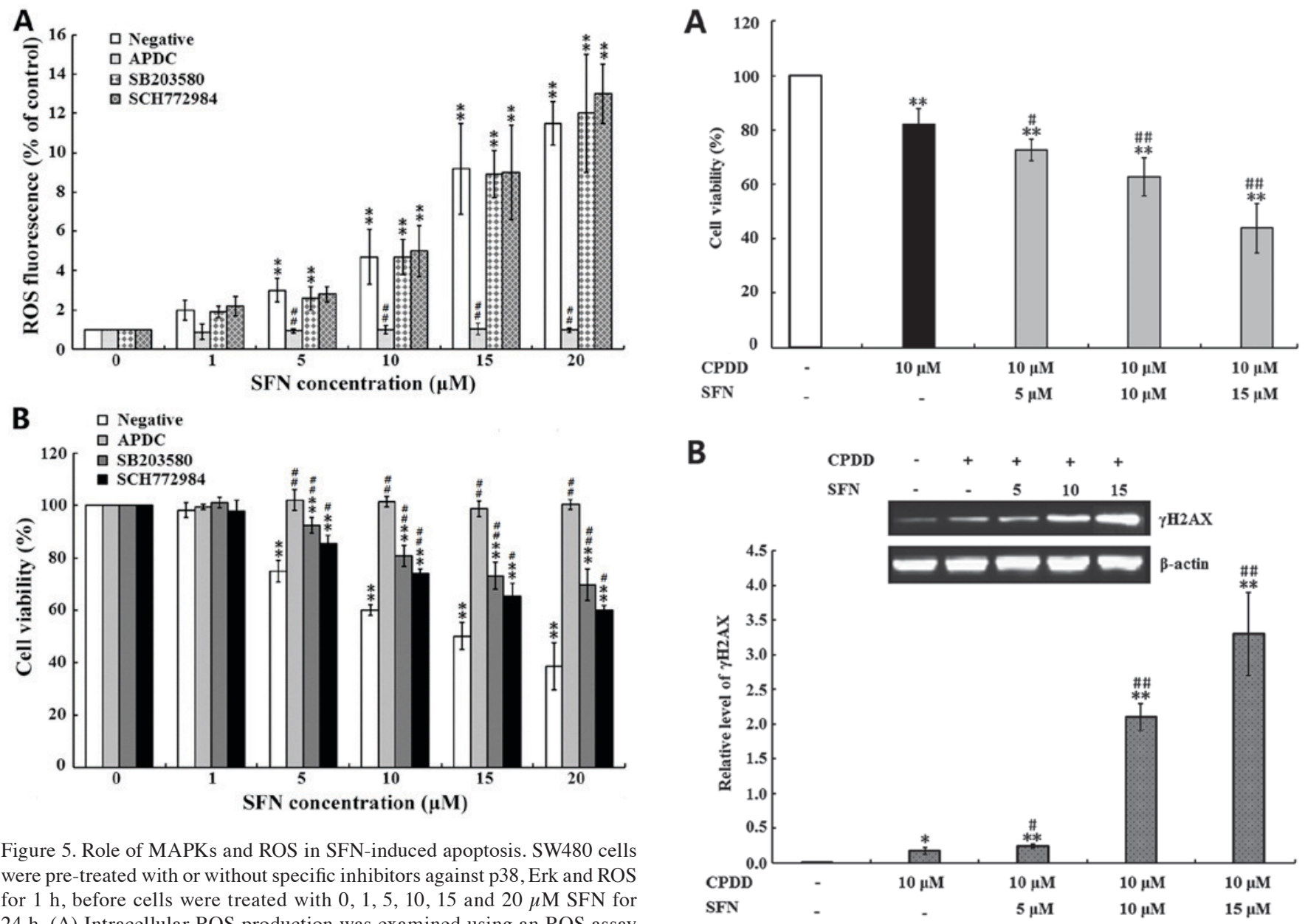

Figure 5. Role of MAPKs and ROS in SFN-induced apoptosis. SW480 cells were pre-treated with or without specific inhibitors against p38, Erk and ROS for $1 \mathrm{~h}$, before cells were treated with $0,1,5,10,15$ and $20 \mu \mathrm{M}$ SFN for 24 h. (A) Intracellular ROS production was examined using an ROS assay kit. (B) Cell viability was assessed using an MTT assay, and the viability of SFN-untreated cells was considered as $100 \%$. For all experiments, the results of three independent experiments are shown. ${ }^{* * *} \mathrm{P}<0.01$ vs. $0 \mu \mathrm{M} \mathrm{SFN} ;{ }^{*} \mathrm{P}<0.05$ and ${ }^{\# \#} \mathrm{P}<0.01$ vs. the negative control. MAPKs, mitogen-activated protein kinases; ROS, reactive oxygen species; SFN, sulforaphane; Erk, extracellular signal-regulated kinases; APDC, ammonium pyrrolidinedithiocarbamate; SB203580, inhibitor of p38 MAPK; SCH772984, inhibitor of Erk.

CPDD-mediated inhibition of HCT-116 cell viability were first determined. As shown in Fig. 6A, treatment with $10 \mu \mathrm{M}$ CPDD alone, and $10 \mu \mathrm{M}$ CPDD combined with 5, 10 and $15 \mu \mathrm{M} \mathrm{SFN}$, significantly inhibited HCT-116 cell viability when compared with the untreated control group $(\mathrm{P}<0.01)$. In addition, when compared with $10 \mu \mathrm{M}$ CPDD treatment alone, combined SFN and CDDP treatment significantly reduced the viability of HCT-116 cells $(\mathrm{P}<0.05$ and $\mathrm{P}<0.01 ;$ Fig. $6 \mathrm{~A})$. The anti-cancer activity of CPDD is based on its interactions with DNA and the subsequent inhibition of DNA replication, which leads to DNA damage (41). Therefore, the present study investigated the effect of SFN on CPDD-induced DNA damage by detecting the levels of $\gamma \mathrm{H} 2 \mathrm{AX}$, a biomarker of DNA damage (42). As shown in Fig. $6 \mathrm{~B}$, treatment with $10 \mu \mathrm{M}$ CPDD alone, or $10 \mu \mathrm{M}$ CPDD combined with 5, 10 and $15 \mu \mathrm{M}$ SFN, significantly increased the level of $\gamma \mathrm{H} 2 \mathrm{AX}$ when compared with the untreated group $(\mathrm{P}<0.05$ and $\mathrm{P}<0.01)$. In addition, combined treatment with SFN and CDDP induced a significant increase in $\gamma \mathrm{H} 2 \mathrm{AX}$ expression when compared with CPDD treatment alone $(\mathrm{P}<0.05$ and $\mathrm{P}<0.01$; Fig. $6 \mathrm{~B})$, which suggested that $\mathrm{SFN}$ may increase the cytotoxic effects of CDDP by increasing the production of $\gamma \mathrm{H} 2 \mathrm{AX}$.

Figure 6. Effect of SFN on CPDD-mediated inhibition of HCT-116 cell viability and $\gamma \mathrm{H} 2 \mathrm{AX}$ expression. (A) HCT-116 cells were treated with $0,1,5$, 10 or $15 \mu \mathrm{M} \mathrm{SFN}$, together with 0 or $10 \mu \mathrm{M}$ CPDD, for $24 \mathrm{~h}$. Cell viability was then assessed using an MTT assay, and the viability of cells without SFN and CPDD treatment were considered as $100 \%$. The results of three independent experiments are shown. (B) The expression of $\gamma \mathrm{H} 2 \mathrm{AX}$ was determined by western blotting, and the quantified results are presented below the images of the protein bands. The bands labeled with '+ CPDD' indicate treatment with $10 \mu \mathrm{M}$ CPDD. $\gamma \mathrm{H} 2 \mathrm{AX}$ expression was normalized to b-actin. ${ }^{*} \mathrm{P}<0.05$ and ${ }^{* * *} \mathrm{P}<0.01$ vs. $0 \mu \mathrm{M}$ SFN plus $0 \mu \mathrm{M}$ CPDD-treated group; ${ }^{* \mathrm{P}}<0.05$ and ${ }^{\# /} \mathrm{P}<0.01$ vs. $10 \mu \mathrm{M}$ CPDD only-treated grouSFN, sulforaphane; CPDD, cisplatin.

\section{Discussion}

SFN is one of the most commonly studied ITCs, and has been reported to inhibit the proliferation of different types of cancer cells by inducing apoptosis $(43,44)$. In agreement with previous studies, the results of the present study revealed that SFN at concentrations between 5 and $20 \mu \mathrm{M}$ significantly inhibited the viability of SW480 cells following exposure for 3, 6, 12, 24 and $48 \mathrm{~h}$. These results were confirmed by flow cytometry analysis, which demonstrated that SFN at concentrations between 5 and $20 \mu \mathrm{M}$ increased apoptosis in SW480 cells following treatment for $24 \mathrm{~h}$. In addition, SFN was observed to induce apoptosis in SW480 cells by increasing ROS generation and the activation of Erk and p38 MAPKs.

ROS are byproducts of aerobic metabolic events that include superoxide $\mathrm{O}^{2-}$, peroxide $\mathrm{H}_{2} \mathrm{O}_{2}$ and hydroxyl $\mathrm{OH}^{-}$radicals (43). In the present study, SFN disturbed the homeostatic balance between the generation and elimination of ROS, leading 
to the accumulation of ROS. MDA, SOD and GSH-Px are important biomarkers of oxidative damage (45). Exposure to SFN increased MDA production in a dose-dependent manner; however, it did not alter the activities of the anti-oxidative enzymes, SOD and GSH-Px. The observed alterations in the levels of these biological parameters indicated that severe oxidative damage was induced by SFN exposure.

In mammalian cells, ROS serve as second messengers to mediate diverse redox-sensitive signaling pathways, such as the MAPK and p53 pathways (46). MAPKs are a family of serine/threonine kinases that produce intracellular signals in response to various stimuli (47). The oxidative stress-dependent activation of MAPKs has been demonstrated to be involved in the induction of cell apoptosis (35). Similar to the results of previous studies, the present study demonstrated that SFN exposure significantly increased the expression levels of p-p38 and p-ERK; however, exposure did not alter the expression levels of the p53 family member, p73. These results suggested that MAPKs were activated by SFN treatment. The roles of ROS, p-Erk and p-p38 in SFN-mediated inhibition of SW480 cell viability were then determined. SW480 cells were pretreated with APDC, SB203580 or SCH772984, which are selective inhibitors of ROS, p38 and Erk, respectively, prior to treatment with SFN. It was revealed that SB203580 and SCH772984 partially alleviated the inhibitory effects of SFN on cell viability. Notably, the ROS inhibitor, APDC, completely attenuated the SFN-mediated reduction in SW480 cell viability. These results indicated that a positive interaction between ROS generation and cell apoptosis may exist, and that this ROS-mediated cell apoptosis may be involved in the activation of p-Erk and p-p38. However, the detailed interactions between ROS production and p-Erk/p-P38 activation requires further investigation in future studies.

Bcl-2 family members are known to be important gatekeepers of apoptotic responses (48-50). Bcl-2 family members are classified into the following three functional groups: Anti-apoptotic factors, such as Bcl-2; pro-apoptotic factors, such as Bax; and pro-apoptotic activators, such as NOXA and PUMA (50,51). Bcl-2 binds and interacts with Bax to prevent mitochondrial pore formation, which subsequently inhibits the execution of cell apoptosis. Therefore, the Bax/Bcl-2 ratio may be used to determine response to therapy and apoptosis (52). The pro-apoptotic activators, which contain a single BH3 domain, are downstream mediators of p53- and p73-dependent apoptosis pathways (53). In the present study, exposure of SW480 cells to SFN did not increase NOXA and PUMA expression, which may be attributed to the deficiencies in p53 and p73 function in SW480 cells. However, SFN treatment significantly inhibited Bcl-2 expression, which led to an increase in the $\mathrm{Bax} / \mathrm{Bcl}-2$ ratio and cell apoptosis. These results were further confirmed by the observed decrease in MM However, the detailed mechanisms by which these cell death signals produced during SFN-induced apoptosis increase the expression of $\mathrm{Bcl}-2$ remains to be elucidated. Previous studies have suggested that the anti-apoptotic functions of Bcl-2 are inhibited by $\mathrm{p} 38 \mathrm{MAPK}$ via phosphorylation $(54,55)$. Therefore, it is possible that $\mathrm{Bcl}-2$ is a mediator that connects the activation of MAPKs to SFN-induced apoptosis.

CPDD is a typical alkylating agent that demonstrates cytotoxic activities against human cancers in vitro. CPDD-based chemotherapy is one of the most important chemotherapeutic treatments available for patients with colon cancer. However, CPDD often produces severe side effects, which limit its efficacy (56). Therefore, there is an urgent requirement to identify more effective agents as cancer therapies. Combination therapy with multiple drugs or modalities is a common treatment strategy for patients with cancer, as it may achieve greater therapeutic benefits when compared with a single drug or modality. In the present study, the effects of SFN exposure on p53-proficient HCT-166 cells in response to CPDD-induced apoptosis were further examined. It was demonstrated that exposure to SFN, even at a low concentration $(5 \mu \mathrm{M})$, increased the sensitivity of HCT-116 cells to the $10 \mu \mathrm{M}$ CPDD-induced reduction in cell viability, potentially via an increase in $\gamma \mathrm{H} 2 \mathrm{AX}$ expression. These results suggest that SFN may be effective as an adjuvant therapy for patients with colon cancer undergoing treatment with CPDD.

In conclusion, the present study successfully established an in vitro colon cancer model of p53-independent, SFN-induced apoptosis. The preliminary results demonstrated that SFN-induced apoptosis in this model may be associated with activation of an intrinsic apoptotic signaling pathway, and that accumulation of ROS is a key event. These observations provide a mechanistic insight into the therapeutic potential of SFN as a clinical treatment for patients with p53-deficient colon cancers. However, further studies are required to confirm the effects of SFN-induced apoptosis in vivo.

\section{References}

1. Bauman JE, Zang Y, Sen M, Li C, Wang L, Egner PA, Fahey JW, Normolle DP, Grandis JR, Kensler TW and Johnson DE: Prevention of carcinogen-induced oral cancer by sulforaphane. Cancer Prev Res (Phila) 9: 547-557, 2016.

2. Amjad AI, Parikh RA, Appleman LJ, Hahm ER, Singh K and Singh SV: Broccoli-derived sulforaphane and chemoprevention of prostate cancer: From bench to bedside. Curr Pharmacol Rep 1: 382-390, 2015.

3. Traka MH, Melchini A and Mithen RF: Sulforaphane and prostate cancer interception. Drug Discov Today 19: 1488-1492, 2014.

4. Folkard DL, Marlow G, Mithen RF and Ferguson LR: Effect of Sulforaphane on NOD2 via NF-кB: Implications for Crohn's disease. J Inflamm (Lond) 12: 6, 2015.

5. Sun CC, Li SJ, Yang CL, Xue RL, Xi YY, Wang L, Zhao QL and Li DJ: Sulforaphane attenuates muscle inflammation in dystrophin-deficient mdx mice via NF-E2-related factor 2 (Nrf2)-mediated Inhibition of NF- $\kappa \mathrm{B}$ signaling pathway. J Biol Chem 290: 17784-17795, 2015.

6. Jang $\mathrm{M}$ and Cho IH: Sulforaphane ameliorates 3-nitropropionic acid-induced striatal toxicity by activating the keap1-Nrf2-ARE pathway and Inhibiting the MAPKs and NF- $\kappa \mathrm{B}$ pathways. Mol Neurobiol 53: 2619-2635, 2016.

7. Tammali R, Reddy AB, Saxena A, Rychahou PG,Evers BM, Qiu S, Awasthi S, Ramana KV and Srivastava SK: Inhibition of aldose reductase prevents colon cancer metastasis. Carcinogenesis 32: 1259-1267, 2011

8. Coriat R, Marut W, Leconte M, Ba LB, Vienne A, Chéreau C, Alexandre J, Weill B, Doering M, Jacob C, et al: The organotelluride catalyst LAB027 prevents colon cancer growth in the mice. Cell Death Dis 2: e191, 2011.

9. Hu T, Wang L, Zhang L, Lu L, Shen J, Chan RL, Li M, Wu WK, To KK and Cho CH: Sensitivity of apoptosis-resistant colon cancer cells to tanshinones is mediated by autophagic cell death and p53-independent cytotoxicity. Phytomedicine 22: 536-544, 2015.

10. Acedo P and Zawacka-Pankau J: p53 family members-important messengers in cell death signaling in photodynamic therapy of cancer? Photochem Photobiol Sci 14: 1390-1396, 2015.

11. Basu S and Murphy ME: p53 family members regulate cancer stem cells. Cell Cycle 15: 1403-1404, 2016. 
12. Pflaum J, Schlosser S and Müller M: p53 family and cellular stress responses in cancer. Front Oncol 4: 285, 2014.

13. Tu HC, Ren D, Wang GX, Chen DY, Westergard TD, Kim H, Sasagawa S, Hsieh JJ and Cheng EH: The p53-cathepsin axis cooperates with ROS to activate programmed necrotic death upon DNA damage. Proc Natl Acad Sci USA 106: 1093-1098, 2009.

14. Wiseman A: $\mathrm{p} 53$ protein or BID protein select the route to either apoptosis (programmed cell death) or to cell cycle arrest opposing carcinogenesis after DNA damage by ROS. Med Hypotheses 67 : 296-299, 2006

15. Yang Y, Jiang L, She Y, Chen M, Li Q, Yang G, Geng C, Tang L, Zhong L, Jiang L and Liu X: Olaquindox induces DNA damage via the lysosomal and mitochondrial pathway involving ROS production and p53 activation in HEK293 cells. Environ Toxicol Pharmacol 40: 792-799, 2015.

16. Dueñas M, Santos M, Aranda JF, Bielza C, Martínez-Cruz AB, Lorz C, Taron M, Ciruelos EM, Rodríguez-Peralto JL, Martín M, et al: Mouse p53-deficient cancer models as platforms for obtaining genomic predictors of human cancer clinical outcomes. PLoS One 7: e42494, 2012.

17. Zhou S, Kachhap S and Singh KK: Mitochondrial impairment in p53-deficient human cancer cells. Mutagenesis 18: 287-292, 2003.

18. Robles AI and Harris CC: Clinical outcomes and correlates of TP53 mutations and cancer. Cold Spring Harb Perspect Biol 2: a001016, 2010

19. Oren M, Tal P and Rotter V: Targeting mutant p53 for cancer therapy. Aging (Albany NY) 8: 1159-1160, 2016

20. Zhang Q, Zeng SX and Lu H: Targeting p53-MDM2-MDMX loop for cancer therapy. Subcell Biochem 85: 281-319, 2014

21. Xiang JF, Wang WQ, Liu L, Xu HX, Wu CT, Yang JX, Qi ZH, Wang YQ, Xu J, Liu C, et al: Mutant p53 determines pancreatic cancer poor prognosis to pancreatectomy through upregulation of cavin-1 in patients with preoperative serum CA19-9 $\geq 1,000 \mathrm{U} / \mathrm{ml}$. Sci Rep 6: 19222, 2016

22. Shi Y, Nikulenkov F, Zawacka-Pankau J, Li H, Gabdoulline R, $\mathrm{Xu}$ J, Eriksson S, Hedström E, Issaeva N, Kel A, et al: ROS-dependent activation of JNK converts p53 into an efficient inhibitor of oncogenes leading to robust apoptosis. Cell Death Differ 21: 612-623, 2014

23. Liu B, Yuan B, Zhang L, Mu W and Wang C: ROS/p38/p53/Puma signaling pathway is involved in emodin-induced apoptosis of human colorectal cancer cells. Int J Clin Exp Med 8: 15413-15422, 2015.

24. Panieri E and Santoro MM: ROS homeostasis and metabolism: A dangerous liason in cancer cells. Cell Death Dis 7: e2253, 2016.

25. Prasad S, Gupta SC and Tyagi AK: Reactive oxygen species (ROS) and cancer: Role of antioxidative nutraceuticals. Cancer Lett 387: 95-105, 2017.

26. Walton EL: The dual role of ROS, antioxidants and autophagy in cancer. Biomed J 39: 89-92, 2016.

27. Nogueira V and Hay N: Molecular pathways: Reactive oxygen species homeostasis in cancer cells and implications for cancer therapy. Clin Cancer Res 19: 4309-4314, 2013.

28. Santabárbara-Ruiz P, López-Santillán M, Martínez-Rodríguez I, Binagui-Casas A, Pérez L, Milán M, Corominas M and Serras F: ROS-Induced JNK and p38 signaling is required for unpaired cytokine activation during Drosophila regeneration. PLoS Genet 11: e1005595, 2015

29. Amin PJ and Shankar BS: Sulforaphane induces ROS mediated induction of NKG2D ligands in human cancer cell lines and enhances susceptibility to NK cell mediated lysis. Life Sci 126: 19-27, 2015.

30. Zhai JW, Gao C, Ma WD, Wang W, Yao LP, Xia XX, Luo M, $\mathrm{Zu} \mathrm{YG}$ and $\mathrm{Fu} \mathrm{YJ}$ : Geraniin induces apoptosis of human breast cancer cells MCF-7 via ROS-mediated stimulation of p38 MAPK. Toxicol Mech Methods 26: 311-318, 2016

31. Youn GS, Lee KW, Choi SY and Park J: Overexpression of HDAC6 induces pro-inflammatory responses by regulating ROS-MAPK-NF- $\mathrm{KB} / \mathrm{AP}-1$ signaling pathways in macrophages. Free Radic Biol Med 97: 14-23, 2016.

32. Wang $\mathrm{H}$, Li D, Hu Z, Zhao S, Zheng Z and Li W: Protective effects of green tea polyphenol against renal injury through ROS-Mediated JNK-MAPK pathway in lead exposed rats. Mol Cells 39: 508-513, 2016

33. Zhang S, Xu R, Luo X, Jiang Z and Shu H: Genome-wide identification and expression analysis of MAPK and MAPKK gene family in Malus domestica. Gene 531: 377-387, 2013.

34. Rhim JH, Luo X, Gao D, Xu X, Zhou T, Li F, Wang P, Wong ST and Xia X: Cell type-dependent Erk-Akt pathway crosstalk regulates the proliferation of fetal neural progenitor cells. Sci Rep 6 26547,2016
35. Liu E, Li J, Shi S, Wang X, Liang T, Wu B and Li Q: Sustained ERK activation-mediated proliferation inhibition of farrerol on human gastric carcinoma cell line by G0/G1-phase cell-cycle arrest. Eur J Cancer Prev 25: 490-499, 2016.

36. Hsu YC, Chang SJ, Wang MY, Chen YL and Huang TY Growth inhibition and apoptosis of neuroblastoma cells through ROS-independent MEK/ERK activation by sulforaphane. Cell Biochem Biophys 66: 765-774, 2013.

37. Chen YJ, Liu WH, Kao PH, Wang JJ and Chang LS: Involvement of p38 MAPK- and JNK-modulated expression of Bcl-2 and Bax in Naja nigricollis CMS-9-induced apoptosis of human leukemia K562 cells. Toxicon 55: 1306-1316, 2010.

38. Smaili SS, Hsu YT, Sanders KM, Russell JT and Youle RJ: Bax translocation to mitochondria subsequent to a rapid loss of mitochondrial membrane potential. Cell Death Differ 8: 909-920, 2001.

39. Benadiba M, Dos Santos RR, Silva Dde O and Colquhoun A: Inhibition of $\mathrm{C} 6$ rat glioma proliferation by [ $\mathrm{Ru} 2 \mathrm{Cl}(\mathrm{Ibp}) 4]$ depends on changes in $\mathrm{p} 21, \mathrm{p} 27, \mathrm{Bax} / \mathrm{Bcl} 2$ ratio and mitochondrial membrane potential. J Inorg Biochem 104: 928-935, 2010.

40. Ray G, Batra S, Shukla NK, Deo S, Raina V, Ashok S and Husain SA: Lipid peroxidation, free radical production and antioxidant status in breast cancer. Breast Cancer Res Treat 59: $163-170,2000$

41. Rebillard A, Lagadic-Gossmann D and Dimanche-Boitrel MT: Cisplatin cytotoxicity: DNA and plasma membrane targets. Curr Med Chem 5: 2656-2663, 2008.

42. Redon CE, Dickey JS, Bonner WM and Sedelnikova OA: $\gamma-\mathrm{H} 2 \mathrm{AX}$ as a biomarker of DNA damage induced by ionizing radiation in human peripheral blood lymphocytes and artificial skin. Adv Space Res 43: 1171-1178, 2009.

43. Buenz EJ: Aloin induces apoptosis in Jurkat cells. Toxicol In Vitro 22: 422-429, 2008

44. Tabolacci C, Rossi S, Lentini A, Provenzano B, Turcano L, Facchiano $\mathrm{F}$ and Beninati S: Aloin enhances cisplatin antineoplastic activity in B16-F10 melanoma cells by transglutaminase-induced differentiation. Amino Acids 44: 293-300, 2013

45. Jing S, Jiang WH and Sun W: Effects of smoking on serum SOD and GSH-PX activities and MDA contents in rats with gastric ulcer. Appl Mech Mater 675-677: 126-129, 2014.

46. Bragado P, Armesilla A, Silva A and Porras A: Apoptosis by cisplatin requires p53 mediated p38alpha MAPK activation through ROS generation. Apoptosis 12: 1733-1742, 2007.

47. Hao W, Wang S and Zhou Z: Tubeimoside-1 (TBMS1) inhibits lung cancer cell growth and induces cells apoptosis through activation of MAPK-JNK pathway. Int J Clin Exp Pathol 8 12075-12083, 2015.

48. Bao FK: The bcl-2 gene family, an important regulator of apoptosis. Sheng Li Ke Xue Jin Zhan 27: 67-69, 1996 (In Chinese).

49. Wu Y and Tang L: Bcl-2 family proteins regulate apoptosis and epithelial to mesenchymal transition by calcium signals. Curr Pharm Des 22: 4700-4704, 2016.

50. Carpio MA, Michaud M, Zhou W, Fisher JK, Walensky LD and Katz SG: BCL-2 family member BOK promotes apoptosis in response to endoplasmic reticulum stress. Proc Natl Acad Sci USA 112: 7201-7206, 2015.

51. Gallenne T, Gautier F, Oliver L, Hervouet E, Noël B, Hickman JA, Geneste O, Cartron PF, Vallette FM, Manon S and Juin P: Bax activation by the $\mathrm{BH} 3$-only protein Puma promotes cell dependence on antiapoptotic Bcl-2 family members. J Cell Biol 185: 279-290, 2009

52. Cory S, Huang DC and Adams JM: The Bcl-2 family: Roles in cell survival and oncogenesis. Oncogene 22: 8590-8607, 2003.

53. Melino G, Bernassola F, Ranalli M, Yee K, Zong WX, Corazzari M, Knight RA, Green DR, Thompson $\mathrm{C}$ and Vousden KH: p73 Induces apoptosis via PUMA transactivation and Bax mitochondrial translocation. J Biol Chem 279: 8076-8083, 2004.

54. Haddad JJ: The role of Bax/Bcl-2 and pro-caspase peptides in hypoxia/reperfusion-dependent regulation of MAPK(ERK): Discordant proteomic effect of MAPK(p38). Protein Pept Lett 14: 361-371, 2007.

55. Pan MH, Chiou YS, Cheng AC, Bai N, Lo CY, Tan D and Ho CT: Involvement of MAPK, Bcl-2 family, cytochrome $\mathrm{c}$, and caspases in induction of apoptosis by 1,6-O,O-diacetylbritannilactone in human leukemia cells. Mol Nutr Food Res 51: 229-238, 2007.

56. He G, He G, Zhou R, Pi Z, Zhu T, Jiang L and Xie Y: Enhancement of cisplatin-induced colon cancer cells apoptosis by shikonin, a natural inducer of ROS in vitro and in vivo. Biochem Biophys Res Commun 469: 1075-1082, 2016 